\title{
Pentastarch resuscitation in severe sepsis and septic shock
}

\author{
Reviewed by: Robert S. Green, BSc, MD; ${ }^{*}$ Peter J. Zed, BSc, BSc(Pharm), PharmD; ${ }^{\dagger}$ \\ Lauralyn Mclntyre, MSc, MD
}

\section{Clinical question}

Does resuscitation with pentastarch reduce mortality when compared with resuscitation using Ringer lactate in patients with severe sepsis or septic shock?

Article chosen

Brunkhorst FM, Engel C, Bloos F, et al. Intensive insulin therapy and pentastarch resuscitation in severe sepsis. N Engl J Med 2008;358:125-39. (The VISEP trial)

\section{Study objective}

The authors of this study sought to assess the safety and efficacy of intensive insulin therapy compared with conventional insulin therapy in patients with severe sepsis or septic shock. In addition, they evaluated the safety and efficacy of hydroxyethyl starch (HES) compared with Ringer lactate in patients in the same patient population. We will discuss only the comparison of HES with Ringer lactate.

\section{BACKGROUND}

Few data are available to guide the choice of colloid or crystalloid for fluid resuscitation in patients with severe sepsis or septic shock. ${ }^{1,2}$ A large randomized controlled trial of $4 \%$ albumin versus $0.9 \%$ saline in a heterogeneous group of critically ill patients found no differences between the solutions, although a subgroup analysis suggested a potential benefit of albumin use in severely septic patients. ${ }^{3}$ Hydroxyethyl starch (HES) solutions are an alterative colloid solution, and have been increasingly used for intravascular volume resuscitation. ${ }^{4}$ However, definitive clinical evidence to support the use of HES solutions in severe sepsis or septic shock is lacking.

\section{STUDY DESIGN AND PATIENT POPULATION}

The study was a prospective, multicentred, randomized, open-label trial with a $2 \times 2$ factorial design. Patients were recruited from multidisciplinary intensive care units (ICUs) at 18 academic tertiary care hospitals in Germany. Patients 18 years of age or older with severe sepsis or septic shock were eligible for enrolment. Patients were eligible for inclusion if the onset of severe sepsis or septic shock was less than 24 hours before admission to the ICU, or less than 12 hours after admission if the condition developed in the ICU. For the purpose of outcome measurement, the treatment period ended 21 days after randomization, or at discharge from the ICU or at the time of death. Patients were ineligible for inclusion if they

- received more than $1000 \mathrm{~mL}$ of HES in the 24 hours before randomization;

- had pre-existing renal failure requiring dialysis or a serum creatinine greater than $320 \mu \mathrm{mol} / \mathrm{L}$;

- required an inspired oxygen fraction greater than 0.7 ;

- had an intracerebral hemorrhage;

- had New York Heart Association class IV heart failure;

- had immunosuppression with cytotoxic chemotherapy; or

- had AIDS or were taking high-dose steroids.

Patients were randomly assigned to receive $10 \%$ pentastarch, a low molecular weight HES or Ringer lactate. During the 96 hours after randomization, a resuscitation protocol was employed to achieve a central venous pressure of $8 \mathrm{~mm} \mathrm{Hg}$. If, during that period, the mean

From the *Departments of Medicine (Division of Critical Care Medicine) and Emergency Medicine, Dalhousie University, Halifax, NS, the tDepartments of Emergency Medicine and Pharmacy, Queen Elizabeth II Health Sciences Centre, Halifax, NS, the ¥Department of Medicine (Division of Critical Care), Ottawa Health Research Institute Centre for Transfusion and Critical Care Research, Ottawa Hospital, Ottawa, Ont.

Submitted Sep. 10, 2008; Accepted May 7, 2009

This article has not been peer reviewed. 
arterial pressure was less than $70 \mathrm{~mm} \mathrm{Hg}$, and mixed venous oxygen saturation was under $70 \%$, the treating physician decided on further measures (fluid repletion, vasopressors and/or inotropes) to raise mean arterial pressure and mixed venous oxygen saturation to prespecified ranges. Decisions on further fluid resuscitation for sepsis-related volume depletion after 96 hours was left to the discretion of the treating physician; however, the study arm assignment resulted in some stipulations in this regard. In the HES group, HES was given until a limit of $20 \mathrm{~mL} / \mathrm{kg} / \mathrm{d}$, then, preferentially, Ringer lactate or other noncolloid fluid was to be administered after this threshold was reached. In the Ringer lactate group, only additional Ringer lactate could be administered.

\section{OUTCOME MEASURES}

The co-primary outcomes were death from any cause at 28 days and morbidity as measured during the intervention by the mean score on the Sequential Organ Failure Assessment (SOFA). ${ }^{5}$ Secondary outcomes included acute renal failure (defined as a doubling of the baseline serum creatinine level or need for renal replacement therapy), time to hemodynamic stabilization, use of vasopressor therapy, mean SOFA subscores, red-cell transfusions, duration of mechanical ventilation, length of ICU stay and 90-day mortality rate.

\section{RESULTS}

After a preplanned interim analysis of 600 patients, the Data and Safety Monitoring Board terminated the trial because of an increased rate of acute renal failure and a trend toward greater 90-day mortality among patients who received HES.

Among the 537 patients who could be evaluated, there was a higher rate of acute renal failure $(34.9 \%$ v. $22.8 \%$, absolute risk increase [ART] $12.1 \%$, number needed to harm $[\mathrm{NNH}] 8, p=0.009$ ), and an increased requirement for renal replacement therapy (31\% v. $18.8 \%$, ARI $12.2 \%, \mathrm{NNH} 8, p=0.001$ ) in the HES group. The rate of death at 28 days was not different between the HES and the Ringer lactate groups $(26.7 \%$ v. $24.1 \%, p=$ $0.48)$. However, there was a trend toward a higher rate of death at 90 days in the HES group $(41.0 \%$ v. $33.9 \%$, $p=0.09)$. The adverse effects appeared to be doserelated, as patients who received higher doses of HES (> $22 \mathrm{~mL} / \mathrm{kg}$ for at least 1 day) had a higher 90-day mortality when compared with patients who received lower doses $(<22 \mathrm{~mL} / \mathrm{kg})(59.6 \%$ v. $30.9 \%$, ARI $28.7 \%, \mathrm{NNH} 3$, $p<0.001)$. The mean SOFA scores did not differ significantly between the HES group and the Ringer lactate group $(8.0$ v. $7.5, p=0.16)$. For patients in the intensive insulin group, there was a trend toward an interaction with HES, based on the renal SOFA score (odds ratio $2.65,95 \%$ confidence interval 1.51 to $4.68, p=0.06$ ) suggesting that the combination of HES and intensive insulin control may affect renal dysfunction in a manner that was not anticipated before the study design. Patients in the HES group also had a lower median platelet count $(p<0.001)$ and received more units of packed red blood cells $(p<0.001)$.

\section{COMMENTARY}

Severe sepsis and septic shock are commonly encountered in emergency departments (EDs), and are associated with high patient mortality., ${ }^{4,6-8}$ Optimal management includes early recognition, broad spectrum antimicrobial administration and aggressive resuscitation. ${ }^{9,10}$ Rapid intravenous fluid administration is a resuscitation priority to correct hemodynamic instability and tissue hypoperfusion associated with these conditions.

Various fluid solutions are available in the ED for the restoration of intravascular volume. ${ }^{11}$ Traditionally, fluids have been classified as crystalloids $(0.9 \%$ saline, Ringer lactate) or colloids (albumin, HES, dextrans or gel solutions). The relative merits of each class of solution have been debated for decades. ${ }^{12-14}$ Systematic reviews on resuscitation fluids have resulted in conflicting conclusions. ${ }^{1,2,15-18}$ Recently, a prospective, randomized, double-blind study of 6997 patients requiring intravascular volume resuscitation in an intensive care setting compared normal saline and $4 \%$ albumin (the Saline Versus Albumin Fluid Evaluation [SAFE] trial). The use of $4 \%$ albumin was found to have no benefit over $0.9 \%$ saline, as there was no difference in 28 -day mortality, ICU length of stay or hospital length of stay. ${ }^{3}$

Hydroxyethyl starch solutions were developed as an alternative colloid for intravascular volume expansion, and are now the dominant colloid used for resuscitating critically ill patients. ${ }^{19,20}$ Synthetic HES solutions are modified polysaccharides and are similar to human glycogen. ${ }^{19}$ Numerous HES fluids are available and possess different physiochemical characteristics based on molecular weight, molar substitution, and the ratio of hydroxyethyl substitution at the $\mathrm{C} 2$ or C6 position (C2:C6 ratio). ${ }^{11,19,21}$ Currently, 3 HES solutions (Pentaspan, Voluven and Hespan) are available in Canada, although little data are available of their use in the ED. 
Concerns with the administration of HES have been voiced by various authors. ${ }^{2,10,12,18,21}$ Recently, adverse events associated with HES use has been increasingly documented in the literature. Adverse events associated with HES include coagulopathy, renal failure, peripheral tissue deposition resulting in puritis, hyperamylsasmia, anaphylactoid reactions and neurologic dysfunction. ${ }^{11}$

The Volume Substitution and Insulin Therapy in Severe Sepsis (VISEP) trial is important to emergency physicians, as the results raise concerns about the use of HES solutions in patients with severe sepsis or septic shock. Patients resuscitated with HES solutions had more acute renal failure, a greater need for renal replacement therapy and a trend toward increased 90-day mortality. In addition, there seems to be a dose-response relationship, as patients who received higher doses of HES had a greater 90-day mortality rate compared with patients who received lower doses. This trial was terminated early for predetermined safety reasons based on harm deemed attributable to the use of HES. The results of the VISEP trial build on the growing body of evidence from randomized controlled trials that suggests these products are harmful in the setting of sepsis. ${ }^{22}$

A balanced appraisal of this study must consider potential limitations before a change in Canadian emergency medicine (EM) use of HES can be advocated. Patients enrolled in the VISEP study were recruited from the ICU, and therefore may differ from ED patients with severe sepsis or septic shock. Although the groups had similar baseline characteristics, the trial was not blinded; hence, it is possible that co-interventions that impacted the results may have differed between the study groups. In addition, $38 \%$ of patients in the HES group exceeded the daily HES dose limit by $10 \%$ or more, and this occurred within the initial 24 hours $75 \%$ of the time, an occurrence that may not reflect current Canadian EM fluid resuscitation practices.

One potentially important consideration of the VISEP study is its factorial design, which allows for individual treatment effects to be evaluated for more than 1 intervention in a single trial. In this study, patients were essentially randomly assigned to groups twice; first to the HES or Ringer lactate group and then to a second "tight" or "conventional" glucose control group. The benefits of factorial design trials include cost savings when compared with the funding required to complete separate studies and reduced sample size requirements. However, the potential for interactions between the study interventions in factorial design trials is an important limitation that must be carefully considered.
Interactions between trial interventions may result in an erroneous inflation or reduction of the treatment effect estimate of either intervention. The investigators of the VISEP trial found a trend toward an interaction in patients who received intensive insulin therapy and HES, as compared with intensive insulin therapy and Ringer lactate, based on the renal SOFA score. This interaction means the independent harm effect associated with HES in the severe sepsis and septic shock setting is potentially lower than estimated in the study, especially since a randomized controlled trial of tight versus conservative insulin control in the critically ill found tight control was associated with an increase in 90-day mortality. ${ }^{23}$

So where does this leave the Canadian emergency physician caring for a critically ill patient with septic shock who requires immediate resuscitation? The VISEP trial does not support the use of pentastarch for the early treatment in this patient population, and therefore alternative resuscitation fluids should be administered. Should these results be generalized to the use of other HES solutions in severe sepsis or septic shock? Little evidence is available to support the benefit of HES solution use in the EM setting. However, in severe sepsis or septic shock, the VISEP trial has demonstrated concerning adverse events in patients. Until further clinical trials with definitive clinical outcomes clearly determine the safety and efficacy of other HES solutions, emergency physicians should avoid their use in patients with severe sepsis or septic shock.

\section{CONCLUSION}

Pentastarch use in patients with severe sepsis or septic shock has been associated with increases in renal failure and, possibly, mortality. Although other HES solutions are available in Canada, unless future research demonstrates safety and improved efficacy in severely septic ED patients, crystalloids should remain the solution of choice for this population.

\section{Competing interests: None declared.}

Keywords: sepsis, resuscitation, pentastarch, factorial clinical trials

\section{REFERENCES}

1. Human albumin administration in critically ill patients: systematic review of randomised controlled trials. Cochrane Injuries Group Albumin Reviewers. BMJ 1998;317:235-40. 
2. Alderson P, Bunn F, Lefebvre C, et al. Human albumin solution for resuscitation and volume expansion in critically ill patients. Cochrane Database Syst Rev. 2004;(4):CD001208.

3. Finfer S, Bellomo R, Boyce N, et al. A comparison of albumin and saline for fluid resuscitation in the intensive care unit. N Engl 7 Med 2004;350:2247-56.

4. McIntyre LA, Fergusson D, Cook DJ, et al. Resuscitating patients with early severe sepsis: a Canadian multicentre observational study. Can J Anaesth 2007;54:790-8.

5. Vincent JL, Moreno R, Takala J, et al. The SOFA (sepsisrelated organ failure assessment) score to describe organ/ dysfunction/failure. On behalf of the Working Group on sepsis-related problems of the European Society of Intensive Care Medicine. Intensive Care Med 1996;22:707-10.

6. Strehlow MC, Emond SD, Shapiro NI, et al. National study of emergency department visits for sepsis, 1992 to 2001. Ann Emerg Med. 2006;48:326-31.

7. Martin GS, Mannino DM, Eaton S, et al. The epidemiology of sepsis in the United States from 1979 through 2000. N Engl 7 Med 2003;348:1546-54.

8. Angus DC, Linde-Zwirble WT, Lidicker J, et al. Epidemiology of severe sepsis in the United States: analysis of incidence, outcome, and associated costs of care. Crit Care Med 2001; 29:1303-10.

9. Green RS, Djogovic D, Gray S, et al. Canadian Association of Emergency Physicians Sepsis Guidelines: the optimal management of severe sepsis in Canadian emergency departments. CJEM 2008;10:443-59.

10. Dellinger RP, Levy MM, Carlet JM, et al. Surviving Sepsis Campaign: international guidelines for management of severe sepsis and septic shock: 2008. Crit Care Med 2008;36: 296-327.

11. Green RS, Hall RI. Con: starches are not preferable to albumin during cardiac surgery: a contrary opinion. I Cardiothorac Vasc Anesth 2008;22:485-91.

12. Traylor RJ, Pearl RG. Crystalloid versus colloid versus colloid: all colloids are not created equal. Anesth Analg 1996;83: 209-12.
13. Imm A, Carlson RW. Fluid resuscitation in circulatory shock. Crit Care Clin 1993;9:313-33.

14. Ross AD, Angaran DM. Colloids vs. crystalloids - a continuing controversy. Drug Intell Clin Pharm 1984;18:202-12.

15. Vincent JL, Navickis RJ, Wilkes MM. Morbidity in hospitalized patients receiving human albumin: a meta-analysis of randomized, controlled trials. Crit Care Med 2004;32:2029-2038.

16. Wilkes MM, Navickis RJ. Patient survival after human albumin administration. A meta-analysis of randomized, controlled trials. Ann Intern Med 2001;135:149-64.

17. Haynes GR, Navickis RJ, Wilkes MM. Albumin administration - what is the evidence of clinical benefit? A systematic review of randomized controlled trials. Eur J Anaesthesiol 2003;20:771-93.

18. Choi PT, Yip G, Quinonez LG, et al. Crystalloids vs. colloids in fluid resuscitation: a systematic review. Crit Care Med 1999;27:200-10.

19. Dieterich HJ. Recent developments in European colloid solutions. 7 Trauma 2003;54(Suppl):S26-30.

20. Schortgen F, Deye N, Brochard L. Preferred plasma volume expanders for critically ill patients: results of an international survey. Intensive Care Med 2004;30:2222-9.

21. Boldt J. Volume therapy in cardiac surgery: Are Americans different from Europeans? J Cardiothorac Vasc Anesth 2006; 20:98-105.

22. Schortgen F, Lacherade JC, Bruneel F, et al. Effects of hydroxyethylstarch and gelatin on renal function in severe sepsis: a multicentre randomised study. Lancet 2001;357:911-6.

23. The NICE-SUGAR Study Investigators. Intensive versus conventional glucose control in critically ill patients. $N$ Engl J Med 2009;360:1283-97.

Correspondence to: Dr. Robert Green, Department of Medicine (Division of Critical Care Medicine), Department of Emergency Medicine, Rm. 377 Bethune Building, 1278 Tower Rd., Halifax NS B3H 2Y9; greenrs@dal.ca 